\title{
A demografia ambiental e a emergência dos estudos sobre população e consumo
}

\author{
Leonardo Freire de Mello* \\ Douglas Sathler $^{\star \star}$
}

Nos últimos anos, a Demografia tem dedicado mais atenção às abordagens interdisciplinares sobre a questão ambiental. A incorporação da temática pela Demografia está em sintonia com o que tem sido observado em praticamente todos os campos do conhecimento, diante da nítida valorização das preocupações relacionadas à sustentabilidade no planeta. No centro das principais discussões ambientais da atualidade, os problemas gerados pelos desequilíbrios entre consumo, ambiente e população merecem cada vez mais destaque. Em um primeiro momento, este artigo oferece uma série de reflexões sobre a recente consolidação da demografia ambiental, buscando avaliar os caminhos ainda não trilhados e as possibilidades de crescimento dos estudos sobre população, consumo e ambiente. Em seguida, é apresentada uma revisão das abordagens oferecidas pelas Ciências Sociais sobre o consumo, investigando como estes estudos têm incorporado a questão ambiental. Ao final, explora-se a inserção do tema - consumo e ambiente - na Demografia, revelando a superação de velhos paradigmas e o surgimento de novos desafios. As reflexões presentes neste estudo demonstram que os trabalhos que lançam uma perspectiva demográfica sobre as implicações ambientais do crescimento do consumo esbarram na constante necessidade de desmistificar qualquer associação simplista entre crescimento demográfico e degradação ambiental. Atualmente, a explosão do consumo é, cada vez mais, vista como algo muito mais temido do que o crescimento exagerado da população.

Palavras-chave: População. Ambiente. Consumo.

\footnotetext{
* Centro de Engenharia, Modelagem e Ciências Sociais Aplicadas - CECS, Universidade Federal do ABC - UFABC, São Bernardo do Campo-SP, Brasil (leonardo.mello@ufabc.edu.br).

${ }^{* *}$ Faculdade Interdisciplinar em Humanidades - FIH/Núcleo de Geociências - Nugeo, Universidade Federal dos Vales de Jequitinhonha e Mucuri - UFVJM, Diamantina-MG, Brasil (doug.sathler@gmail.com).
} 


\section{Introdução}

Nas últimas três décadas, as concepções sobre o meio ambiente evoluíram bastante com a multiplicidade de preocupações que passaram a ser incorporadas à temática. $\mathrm{A}$ noção de impacto ambiental amadureceu, não mais se limitando às consequências físicas da atuação humana, passando a considerar, também, os aspectos sociais, econômicos e culturais nas diversas escalas de análise. Se nos anos 1970, sobretudo após a Declaração de Estocolmo (1972), as atenções praticamente restringiram-se aos desafios criados pelo aumento dos níveis de poluição e contaminação, atualmente, os problemas ambientais têm sido pensados de maneira mais ampla.

As Ciências Sociais se atrasaram na incorporação das questões ambientais em seus respectivos temas de pesquisa e apenas na última década os estudos sistemáticos sobre população e ambiente ganharam mais fôlego. Assim, no universo das abordagens interdisciplinares, a participação da Demografia nesse tipo de discussão tem sido crescente, oferecendo novas ferramentas e perspectivas para o tema (FREIRE DE MELLO; HOGAN, 2007; MARANDOLA JR.; HOGAN, 2007; SATHLER, 2012).

As dimensões humanas devem assumir papel de destaque na literatura sobre as mudanças ambientais. 0 ser humano é multidimensional e pode ser estudado no plano individual e familiar, sendo parte de uma população que vive um dado contexto social e cultural. 0 ambiente é multiescalar e as análises devem estar atentas para as transformações ambientais locais, regionais e globais ao longo do tempo (HOGAN et al., 2010). Segundo Hogan et al. (2010, p.14), “a relação entre estas dimensões e escalas expressa a natureza da questão ambiental: uma população busca no ambiente a reprodução e a sustentabilidade de sua vida”.

No centro das principais discussões ambientais da atualidade, os problemas gerados pelos desequilíbrios entre consumo, ambiente e população merecem a atenção das abordagens que privilegiam as dimensões humanas. 0 consumo, e tudo o que gira em torno desta prática, vem se consolidando como importante base de sustentação de um modelo de desenvolvimento hegemônico global (FREIRE DE MELLO; HOGAN, 2007; UNEP, 2010). As Ciências Sociais e a Demografia têm contribuído, cada vez mais, nas discussões sobre os impactos do consumo nas mudanças ambientais contemporâneas.

Diante disso, o presente artigo traz uma discussão sobre população, ambiente e consumo, com base em bibliografia selecionada, tendo em vista a carência na sistematização destes estudos na literatura brasileira recente e objetivando estimular a referida discussão no âmbito da Demografia brasileira, por considerá-la fundamental no momento atual, como evidenciado pelo importante debate sobre o tema entre David Lam e Stan Becker, em número recente da Demography.

A metodologia dessa revisão privilegiou a identificação dos conceitos e debates mais relevantes, com base nas seguintes perguntas de pesquisa: quais são os principais desafios da consolidação de uma demografia ambiental, sobretudo diante das diversas possibilidades analíticas que a Demografia oferece para a questão ambiental? Quais são 
os caminhos ainda não trilhados para a consolidação da demografia ambiental? Qual é a inserção das Ciências Sociais nas discussões sobre consumo e ambiente? Como a Demografia tem contribuído nestes debates? 0 aumento populacional necessariamente implica aumento imediato do consumo no planeta?

Assim, o primeiro tópico explora um conjunto de textos selecionados, privilegiando - mas não restrito apenas a - a produção brasileira recente, que discutem como a demografia ambiental, apesar de todas as dificuldades e dos caminhos ainda não trilhados, tem sido um campo fértil para o desenvolvimento de estudos sobre população, consumo e ambiente nos círculos acadêmicos. Também é apresentada uma revisão das abordagens das Ciências Sociais sobre o consumo, investigando como estes estudos têm incorporado a questão ambiental. Ao final, o texto explora a inserção do tema - consumo e ambiente - na Demografia, revelando a superação de velhos paradigmas e o surgimento de novos desafios. Tendo em vista o caráter interdisciplinar do tema, a revisão não se restringiu aos trabalhos publicados dentro da Demografia, buscando contribuições de vários campos do conhecimento que, se bem articulados, enriquecem as discussões e contribuem para a consolidação da demografia ambiental.

\section{População, espaço e ambiente: rumo à consolidação de uma demografia ambiental}

Atualmente, um novo campo dentro da Demografia e dos estudos de população, que Daniel Hogan denominou, no Brasil, de demografia ambiental, está dedicando mais atenção às relações entre população e ambiente. Paralelamente ao aumento significativo do consumo no planeta, os componentes da dinâmica demográfica se comportaram com bastante dinamismo durante o último século em diversas partes do globo. Estas transformações demográficas foram acompanhadas de mudanças ambientais gravíssimas e ainda existe muito para entender sobre as relações entre as variáveis populacionais e o ambiente.

A partir dos anos 1980, a difusão global de casos de contaminação ambiental e suas consequências para a saúde e a vida humana motivaram o desenvolvimento de estudos sobre população e ambiente (HOGAN et al., 2010).

Já na década de 1990, os estudos passaram a incorporar, sobretudo, as preocupações ambientais com o crescente aumento da emissão de gases de efeito estufa e a poluição do ar (BONGAARTS, 1992; BIRDSALL, 1992; O’NEILL et al., 2001), as mudanças na cobertura e no uso do solo e o desmatamento (BILSBORROW; DELARGY, 1991; BILSBORROW; STUPP, 1997) e as relações entre desastres ambientais e a migração (ANDERTON et al. 1994; HUNTER, 1998).

No entanto, a Demografia demorou a incorporar as questões ambientais e apenas recentemente este campo de pesquisa tem se tornado mais abrangente. Na década de 1990, alguns estudos ofereceram evidências que buscaram explicar as dificuldades da Demografia no estabelecimento de uma linha de pesquisa sólida para tratar as questões ambientais (DAVIS, 1991; KEYFITZ, 1992; PEBLEY, 1998). 
Davis (1991) argumenta que muitos pesquisadores investiram elevado tempo em pesquisas voltadas para a criação de mecanismos para frear o crescimento populacional, visto como fator que exerce grande pressão sobre os recursos naturais. Paradoxalmente, também existe o argumento de que as causas centrais dos problemas ambientais não são demográficas, ressaltando a importância das instituições sociais, da eficiência dos mercados, do nível tecnológico e da distribuição de renda.

Por outro lado, Keyfitz (1992) ressalta que o excesso de peso dado a estes temas pode levar muitos cientistas sociais à interpretação errônea de que as questões populacionais têm pouco ou nenhum impacto nas transformações ambientais.

Outro elemento que distancia a Demografia dos estudos ambientais é a dificuldade de diálogo dos estudiosos da população com outras áreas, a exemplo da Biologia, Bioquímica, Agronomia e Climatologia. Como ressalta Pebley (1998), na década de 1990, a carência de dados longitudinais locais para o estudo dos impactos ambientais também agravou esta situação.

As abordagens mais recentes sobre população e ambiente têm incorporado novos aspectos, buscando refletir sobre as relações entre as transformações demográficas (envelhecimento populacional, estrutura etária e distribuição espacial) e questões como qualidade e disponibilidade de água, geração de lixo, biodiversidade, paisagem, desastres naturais, entre outras (RCEP, 2011).

0 trabalho de Hogan et al. (2010) destaca algumas questões emergentes que passaram a ser incorporadas: as transformações na estrutura etária da população e as mudanças na composição domiciliar (menores unidades com maior consumo energético); a cidade como forma de organização espacial da população; população e consumo; e população e ambiente no contexto das mudanças climáticas. Por fim, os autores também destacam que a valorização do espaço nos estudos sobre população e ambiente é uma característica presente nos trabalhos produzidos no país.

Embora haja um aumento nas pesquisas que exploram questões relacionadas aos componentes da dinâmica populacional e as alterações ambientais de maneira isolada, no Brasil os estudos interdisciplinares que buscam investigar a relação entre estes temas ainda são incipientes.

Se, no passado, as discussões em torno do impacto do estoque populacional sobre o ambiente ganharam destaque, atualmente, Harper (2011) revela que ainda existe carência em abordagens que considerem as implicações de outras variáveis demográficas, como densidade populacional e composição da população, sobre o ambiente.

A comunidade científica não pode cessar os esforços em busca do fortalecimento dos estudos sobre população, espaço e ambiente, já que ainda existe muito espaço a conquistar. Diante disso, cabe o seguinte questionamento: quais são os caminhos ainda não percorridos para a consolidação da demografia ambiental?

Existem várias possíveis respostas para esta pergunta, embora a maior parte delas aponte para a necessidade de aprofundamento e consolidação de iniciativas já percebidas na comunidade científica, mesmo que de maneira tímida, por demógrafos e não demógrafos. 
Para que a demografia ambiental se torne mais influente e robusta, deve-se, em primeiro lugar, garantir o seu crescimento dentro da própria Demografia, com a ampliação de abordagens interdisciplinares que envolvam, cada vez mais, a participação de profissionais também de outras áreas do conhecimento. Além disso, espera-se que a demografia ambiental se valorizará quando mais e mais pesquisadores não demógrafos identificarem na Demografia ferramentas importantes para a complementação de suas abordagens, devendo, portanto, ser estimulada dentro e fora do campo tradicional da Demografia.

Este não é um caminho fácil de ser percorrido, embora existam algumas estratégias que mereçam ser discutidas. Cabe aos demógrafos e às associações (lussp, Alap, Abep, PAA, entre outras) trabalhar em prol de uma maior incorporação de análises demográficas nos grandes relatórios sobre mudanças ambientais, sobretudo aqueles realizados pelo IPCC, ONU e Banco Mundial. Isso traria maiores possibilidades de crescimento da demografia ambiental, tendo em vista que estes estudos, de certa forma, servem de referência para toda a comunidade científica internacional, balizando abordagens e indicando campos de pesquisa promissores.

Outra possibilidade é a ampliação do levantamento de dados e informações com base em recortes geográficos mais favoráveis aos estudos sobre população e ambiente, a exemplo de bacias hidrográficas, biomas, domínio morfoclimáticos e áreas de vulnerabilidade socioambiental.

Ainda, cabe reforçar a estruturação de base de dados e garantir a multiplicação de plataformas interativas que permitem a integração de dados espaciais, demográficos e ambientais. As montagens de bancos de dados secundários devem considerar estas possibilidades de integração, o que reduziria boa parte das limitações existentes e, também, a necessidade de esforços para a compatibilização de bases cartográficas.

Ademais, a Demografia deve estar sempre atenta às grandes discussões ambientais globais, a exemplo das possibilidades de adaptação às mudanças climáticas globais e suas repercussões sobre as populações com maior nível de vulnerabilidade. Também não se pode perder de vista que boa parte dos problemas que ameaçam a saúde e a vida acontece localmente ou regionalmente. Se os problemas ambientais contemporâneos passam por um entendimento multiescalar, a demografia ambiental também deverá criar novas possibilidades analíticas em sintonia com a natureza destes problemas.

Estas iniciativas devem ser multiplicadas em prol de uma demografia ambiental mais influente e participativa, que ofereça alternativas mais acuradas para a mitigação e adaptação aos principais problemas ambientais nas suas diversas escalas. Segundo Hogan et al. (2010, p. 96), “já está na hora de assumirmos uma demografia ambiental, que contribua de forma sistemática para a compreensão e construção de um mundo sustentável”. Segundo os autores, "esse é um esforço coletivo para o futuro que agrega à reflexão ambiental um olhar propriamente demográfico". Nesse sentido, as repercussões negativas do consumo estarão presentes nesse esforço constante em busca da consolidação de uma demografia ambiental. 


\section{0 papel das Ciências Sociais nos estudos sobre consumo e ambiente}

0 movimento ambientalista vem sistematicamente ganhando força e encontrando espaço em todas as esferas da vida social (HOGAN et al., 2010).

Se a Rio 92 foi um importante marco para o aumento das preocupações ambientais, nos últimos anos, todos os esforços que culminaram na conferência Rio+20 e no $5 \%$ relatório (AR-5) do International Panel on Climate Change (IPCC, 2013) demonstraram que a sociedade, de forma geral, evoluiu bastante nas discussões ambientais. Nesse contexto, ganham destaque o maior volume de publicações científicas na área e a incorporação dos temas ambientais por outros campos do conhecimento.

A literatura sobre população, consumo e ambiente tem destacado o papel desempenhado pelos estilos de vida e padrões de produção e consumo a eles relacionados, tendo em vista as mudanças ambientais contemporâneas (FREIRE DE MELLO; HOGAN, 2007; FREIRE DE MELLO, 2009).

0 aumento do nível de crítica e questionamento, tanto científico quanto social, sobre o modo de vida centrado na utilização intensiva de combustíveis fósseis parece evidente, uma vez que os modelos de crescimento econômico e de desenvolvimento vigentes têm se mostrado insustentáveis (STERN et al., 1997; PRINCEN et at., 2002; UNEP, 2002; MYERS, 2003; HOLDEN, 2004).

Atualmente, a produção de bens e serviços é extremamente dependente do crescimento da demanda por consumo. A capacidade intelectual e inventiva da espécie humana tenta adequar a natureza às suas crescentes demandas por energia e matérias-primas, para a satisfação de padrões de produção e consumo cada vez mais intensivos, embora não exista equilíbrio nesta relação conflituosa entre homem e natureza. Os estilos de vida e as escolhas deles derivadas impactam diretamente o ambiente, gerando estímulos que provocam a reação dos diferentes setores produtivos para satisfazerem as necessidades e demandas existentes (FREIRE DE MELLO; HOGAN, 2007; FREIRE DE MELLO, 2009).

Durante o século XX, em especial após a Segunda Guerra Mundial, o consumo em massa se desenvolveu e consolidou de forma global, criando e disponibilizando - a custos financeiros decrescentes, mas a custos ambientais e sociais crescentes - uma gama de produtos e serviços jamais experimentada (FREIRE DE MELLO; HOGAN, 2007; FREIRE DE MELLO, 2009; KUMAR, 2013).

0 problema com que nos confrontamos no momento é que estilos de vida e padrões de consumo - e, consequentemente, de produção - com grande potencial de degradação ao ambiente vêm se consolidando como hegemônicos em grande parte das economias e sociedades (KEMPF, 2008). A multiplicação do consumo não estimulou, na mesma proporção, soluções baseadas em formas sustentáveis de interação com os recursos naturais do planeta.

O geógrafo norte-americano Robert Kates recupera uma definição de consumo, desenvolvida pela Royal Society of London em conjunto com a U.S. National Academy of Scien- 
ces no final da década de 1970 que, apesar de já ter mais de 40 anos, aponta caminhos interessantes na busca de um melhor entendimento da questão, associando o consumo humano à transformação de materiais e energia.

Este processo pode gerar desequilíbrios caso o consumo torne os materiais e a energia menos disponíveis para o futuro, ou afete negativamente os sistemas biofísicos de modo a ameaçar a saúde e o bem-estar das populações (FREIRE DE MELLO; HOGAN, 2007). Por sua vez, Kates (2000) esclarece que existem lacunas teóricas e empíricas nos estudos sobre o consumo, diante de dificuldades conceituais básicas e de escassez de fontes de dados (KATES, 2000).

De maneira geral, consumo pode ser entendido como a principal forma de interação entre os seres vivos e o ambiente. Sendo assim, a vida é, em essência, consumo, seja por meio do uso do solo como substrato e fonte de nutrientes, seja pela utilização de combustíveis fósseis como fonte de energia ou pelas demais formas de relacionamento do homem com o ambiente (FREIRE DE MELLO; HOGAN, 2007; FREIRE DE MELLO, 2009).

As diferentes espécies animais e vegetais têm que interagir com o ambiente e os recursos disponíveis para poderem se desenvolver, alterando-os e, até mesmo, destruindo-os completamente ao longo do processo de interação (PATERSON, 2006).

0 consumo por si só não é algo mal e que deve ser combatido, mas, ao se tornar um fim em si mesmo, seja como objetivo principal dos indivíduos ou como medida fundamental do sucesso individual, transforma-se em ameaça forte e agressiva ao ambiente e, consequentemente, aos seres vivos que dele dependem. A consolidação de uma cultura e de um modo de vida baseados essencialmente na capacidade de consumir dos indivíduos é algo que deve fazer soar os alertas (GARDNER et al., 2004).

No século XIX, Marx, Weber e Durkheim abordaram o consumo de forma quase sempre periférica às suas discussões centrais. Segundo Carrabine (2010, p. 50-51), o consumo é concebido, por exemplo, como "uma instância do 'fetichismo da mercadoria' induzido pelo capitalismo na tradição marxista. É entendido como fator de estratificação social baseado em exibições instrumentais de riqueza e prestígio”, na tradição weberiana, ou como consequência do "industrialismo moderno que era o gerador de desejos de consumo patologicamente insaciáveis que corroem a base moral da ordem social”, na tradição durkheimiana.

Entre os autores que contribuíram de forma efetiva para tornar o consumo um verdadeiro campo de conhecimento e pesquisa, é importante destacar Thorstein Veblen, economista e sociólogo norte-americano, conhecido por ser um dos fundadores da New School for Social Research e por popularizar o termo consumo conspícuo no livro The theory of the leisure class, publicado em 1899, que, de certa maneira, orientaria e enviesaria os estudos sobre o tema ao longo dos séculos seguintes e que se baseava na ideia de que os indivíduos utilizam o consumo e a exibição - muitas vezes ostensiva - de bens e posses materiais como forma de "indicar ou realçar o próprio prestígio aos olhos dos demais" (JOHNSON, 1997, p. 51).

Talcott Parsons, sociólogo norte-americano responsável pelo desenvolvimento da teoria do funcionalismo estrutural, defendia a ideia, fortemente baseada em Weber e Durkheim, 
de que sociedade se estrutura em diferentes partes, cada uma com funções próprias e únicas que, coordenadas, promovem a estabilidade social. Assim, a noção de prestígio seria central nos sistemas de estratificação social (PARSONS, 1960). Dessa forma, o consumo conspícuo se materializa, por exemplo, quando indivíduos da chamada, por Veblen, “classe ociosa" demarcam seu posicionamento social superior "demonstrando que possuem tanto que podem dar-se ao luxo de desperdiçar em enorme escala” (JOHNSON, 1997, p. 51).

Esta ideia ainda permeia e influencia fortemente algumas análises contemporâneas sobre consumo e ambiente, que invadiram e ganharam espaço dentro do pensamento sociológico ao longo das décadas de 1980 e 1990. Segundo Johnson (1997, p. 52), “comportamento, gosto e preferências pessoais têm consequências sociais, cujo significado e alcance se situam muitas vezes além da consciência que temos deles".

A emergência e consolidação das questões ambientais observadas ao longo da segunda metade do século XX fizeram com que ocorresse uma mudança significativa na percepção e na abordagem dada pelas Ciências Humanas e Sociais ao tema do consumo. 0 ramo da Sociologia dedicado ao estudo do consumo, ao passar a desafiar algumas das premissas básicas da disciplina, promoveu mudanças nos usos do termo, que passou a não se restringir às conceituações reducionistas e francamente negativas, relacionadas intensamente com as ideias de destruição, desperdício e exaustão.

Esse movimento das Ciências Sociais foi incisivamente marcado por uma série de estudos fundamentais sobre o consumo, ${ }^{1}$ que se contrapunham às ideias fomentadas no século XIX que caracterizavam o consumo como algo negativo, socialmente inútil e que deveria, portanto, ser depreciado, principalmente por ser uma atividade predominantemente feminina (McCRACKEN, 2003).

Coincidentemente, isso ocorreu no mesmo momento histórico da adoção por vários governos ao redor do planeta de políticas neoliberais e da ascensão e consolidação do ideal da liberdade de escolha como a aspiração central da vida individual, social, econômica e política dos indivíduos e das sociedades (CARRABINE, 2010).

A argumentação destes novos teóricos do consumo, segundo Carrabine (2010, p. 50), destacava a necessidade de se abandonar as "antigas preocupações dos teóricos do século XIX, que identificavam a produção industrial e a posição de classe como fonte básica dos propósitos e antagonismos da sociedade", buscando demonstrar, de forma clara e coerente, que são as relações sociais que estruturam "a aparente individualidade de tais escolhas, desejos e significados".

Essa ressignificação do consumo dentro da Sociologia não quer dizer que, antes da década de 1980, os teóricos haviam abordado o tema de uma única forma. Muito pelo contrário, as acepções foram as mais diversas. Entretanto, Carrabine (2010, p. 50) revela que "o tema permaneceu às margens de uma teoria social mais ampla".

\footnotetext{
$\overline{{ }^{1} \text { Ver Featherstone (1995) }}$, Slater (2001) e Seneett (2006).
} 
No entanto, mesmo após duas décadas de evolução nos estudos sobre consumo e ambiente, a socióloga Sara Curran e o geógrafo Alex de Sherbinin (2004) afirmam que, apesar de a questão do consumo estar fortemente conectada às relações população-ambiente, as duas literaturas ainda têm seguido caminhos diferentes, com algumas poucas e significativas exceções (FREIRE DE MELLO; HOGAN, 2007; FREIRE DE MELLO, 2009).

Além disso, os autores identificam três áreas predominantes de pesquisa nas Ciências Sociais sobre a temática consumo/ambiente, com grandes possibilidades de diálogo com a demografia: pesquisas dedicadas à mensuração e análise do consumo no nível de domicílios, nas quais o foco vem passando das estatísticas de consumo nacionalmente agregadas para as análises no nível de domicílio; pesquisas sobre estimativa, cálculo e análise de pegadas ecológicas e contabilidade de fluxos de materiais, que agregam principalmente pesquisadores das áreas biológicas e físicas - com alguma participação de cientistas sociais - e voltadas para o desenvolvimento de índices como a pegada ecológica, procurando demonstrar sua validade e capacidade de predição; e pesquisas direcionadas para análise de valores, atitudes, comportamentos e estilos de vida, majoritariamente conduzidas por cientistas sociais que tentam identificar e entender a influência dos valores e atitudes no comportamento do consumidor, assim como o papel desempenhado pelo consumo nos processos de aumento de consciência ambiental e de mudanças de atitude (CURRAN; DE SHERBININ, 2004, p. 108).

Curran e De Sherbinin (2004) indicam que muitos estudos voltados para mensuração e análise do consumo dos domicílios têm como ponto de partida a utilização da variável "energia per capita", ${ }^{2}$ atribuindo tal popularidade a algumas vantagens que esta métrica possui em relação a outras mais complexas. Ainda, além dos domicílios, existem outras unidades de consumo na sociedade, como, por exemplo, a administração pública e as corporações (CURRAN; DE SHERBININ, 2004).

O segundo grupo de estudos dedica-se à estimativa, ao cálculo e à análise de pegadas ecológicas e contabilidade de fluxos de materiais. 0 termo, cunhado por Mathis Wackernagel e William Rees no final dos anos 1980 (ecological footprint), quantifica, de maneira razoavelmente simples, os recursos naturais necessários para a manutenção de determinado estilo de vida por meio do atendimento às necessidades e às demandas de consumo derivadas deste estilo de vida. Trata-se do somatório de todas as componentes do consumo relacionadas à geração de impactos ambientais (WACKERNAGEL; REES, 1988).

No caso das nações mais industrializadas, boa parte dos materiais necessários à manutenção do status quo não está disponível localmente, o que leva à necessidade da busca e apropriação destes recursos em outras regiões do planeta, expandindo suas pegadas e a amplitude de seus impactos ambientais, levando-a do nível local ou regional para a escala global.

\footnotetext{
${ }^{2}$ Ver OECD (2008)
} 
Curran e De Sherbinin (2004) atribuem a rápida difusão e popularização da análise da pegada ecológica ao fato de ela ser uma metodologia relativamente simples de inventário, contabilidade e incorporação das externalidades (ambientais e sociais) das atividades humanas, bem como por ampliar o foco da análise ao reconhecer e incorporar que não só as atividades produtivas, mas também o comércio e os serviços implicam consumo de recursos e insumos. Os estudos de pegada ecológica, ao apontarem a importância desempenhada pelo comércio global contemporâneo na geração de impacto ambiental, também estimulam práticas mais justas e menos impactantes, a exemplo do fair trade. Segundo Spaargaren (2000a, 2000b, 2003), essas práticas representam uma possibilidade, cada vez mais real e concreta, de mudança dos padrões de produção e consumo.

Os estudos voltados para a contabilidade de fluxos de materiais (material flow accounting - MFA e material and energy flow accounting - MEFA), ${ }^{3}$ nada mais são do que análises do consumo baseadas na premissa de que sustentabilidade é um problema da interação sociedade-natureza ao longo do tempo. Esta abordagem busca traçar os fluxos socioeconômicos de materiais e energia, avaliando as mudanças em processos e padrões relevantes nos ecossistemas relacionados com as intervenções nos sistemas naturais, aumentando a utilidade destes sistemas para os objetivos econômicos. Objetiva responder às seguintes questões: quais mudanças as atividades socioeconômicas causam nos sistemas naturais? Quais forças socioeconômicas estão por trás destas mudanças e como elas podem ser influenciadas? Como as mudanças nos sistemas naturais impactam a sociedade? Como a sociedade pode lidar com as mudanças que ela própria provocou? (HABERL, 2004; FREIRE DE MELLO; HOGAN, 2007).

O terceiro grupo de estudos sobre consumo/ambiente trabalha com análises de valores, atitudes, comportamentos e estilos de vida. ${ }^{4}$ De acordo com esta abordagem, o comportamento do consumidor é determinado por uma série de fatores, tais como valores pessoais, nível educacional e disponibilidade de renda, que interferem tanto nas preferências de consumo dos indivíduos quanto na determinação do grau e da intensidade com que os indivíduos podem efetivamente consumir. De maneira geral, conforme Wilk (1998), os valores individuais variam em função de três causas básicas: diferenças entre gerações; mudanças nas condições durante o ciclo de vida do indivíduo; e influências externas sazonais como eventos pessoais e sociais de grande impacto sobre o indivíduo (FREIRE DE MELLO; HOGAN, 2007; FREIRE DE MELLO, 2009).

Os trabalhos sobre estilo de vida vêm crescendo em número e importância nas análises das relações de consumo. Os estilos de vida podem ser agrupados em clusters baseados em semelhanças e, com isso, oferecer meios para a identificação, por exemplo, dos pontos de alavancagem da mudança que seriam mais relevantes para cada cluster ou dos clusters que produzem os impactos ambientais mais significativos e que, portanto, demandam uma

\footnotetext{
${ }^{3}$ Ver Bringezu (1997), Eurostat (2001), Amann et al. (2002), Hammer e Hubacek (2002) e Hinterberger et al. (2003).

${ }^{4}$ Ver Wilk (1998) e Thorgerson e Olander (2002).
} 
maior e mais rápida intervenção. Uma vez que os clusters de consumo são identificados, políticas podem ser desenvolvidas de acordo com os diferentes modelos ou teorias do consumo (WILK, 2002).

Uma limitação bastante significativa na abordagem do consumo por meio da análise de valores, estilo de vida e cultura relaciona-se com a dificuldade de se identificarem claramente as formas pelas quais preços, rendas e gostos afetam o comportamento do consumidor. A pesquisa na interface população-ambiente, ao enfatizar as conexões entre as pessoas e as mudanças ambientais, pode contribuir significativamente para a obtenção de informações importantes sobre a variabilidade na elasticidade das curvas de demanda, em análises comparativas entre lugares e culturas diferentes (FREIRE DE MELLO, 2009).

As contribuições das Ciências Sociais sobre a relação consumo e ambiente evoluíram, passando a oferecer um arcabouço teórico aplicável também a outras áreas do conhecimento. As ideias levantadas neste tópico podem ser aproveitadas não apenas para discussões mais substantivas dentro da Demografia, mas também para dar suporte teórico para a aplicação de técnicas demográficas e para o uso de ferramentas específicas dos estudos populacionais.

Tendo em vista os diversos parâmetros demográficos e domiciliares inerentes à relação consumo-ambiente, estes estudos oferecem subsídios valiosos para a superação de qualquer visão simplista da relação entre crescimento populacional e degradação ambiental, como será demonstrado no próximo tópico.

\section{População, consumo e ambiente: da bomba populacional à crise do consumo}

A relação entre população e consumo e, consequentemente, a concepção do termo pela Demografia foram fortemente marcadas pela obra clássica Essay on population, de Thomas Malthus (1798), que estabeleceu a base lógica que orientaria a análise demográfica fundamentada na indubitável e determinista conexão entre crescimento populacional exagerado e suas consequências para a humanidade. Conforme as interpretações demográficas do autor no final do século XVIII, o crescimento populacional e a reprodução limitada dos meios de subsistência levariam as sociedades humanas à catástrofe e ao colapso econômico.

A concepção malthusiana traz e consolida, tanto na ciência quanto no senso comum, a ideia de que o crescimento da população impediria o bem-estar geral, sendo a principal causa da pobreza, da miséria e da fome, com o autor propondo algumas soluções para impedir as catástrofes relacionadas à tão temida explosão demográfica, com destaque para a manutenção dos salários em níveis não superiores ao de subsistência, considerando que existiria uma relação direta entre renda e natalidade. No entanto, a transição demográfica nos países mais desenvolvidos, com a queda sistemática da fecundidade, afastou por algumas décadas o "fantasma malthusiano" (ALVES, 2002, 2006).

Muitas transformações econômicas e sociais marcaram o mundo a partir de meados do século XX. O período pós-Segunda Guerra Mundial foi caracterizado por uma significativa 
melhoria das condições de vida entre as sociedades ocidentais, marcado pela busca do Welfare State e pela disseminação de um modo de vida baseado fortemente nos padrões de produção e consumo estabelecidos pelos Estados Unidos e seus aliados (GONÇALVES, 2013).

Parte da tecnologia desenvolvida como esforço para vencer a guerra tornou-se acessível a grandes parcelas das populações destes países, popularizando a utilização de produtos e serviços (antibióticos, viagens aéreas internacionais, serviços de comunicação, entre outros), que rapidamente passaram a fazer parte da vida cotidiana de milhões de pessoas por todo o mundo (MINDELL, 2009).

Assim, houve uma alteração definitiva da relação entre sociedade e ambiente, com a consolidação da sociedade de consumo como paradigma desenvolvimentista. Tais avanços tecnológicos e científicos, especialmente na Medicina e na Química, impactaram de forma direta a dinâmica demográfica dos diversos países. A difusão das tecnologias de controle da mortalidade e a melhoria do padrão de vida das populações aumentaram a expectativa de vida média (WOOD; CARVALHO, 1988). Estas transformações surtiram efeitos também nos países até então rotulados de subdesenvolvidos ou do Terceiro Mundo.

Após meados do século XX, os debates sobre a possibilidade de uma explosão demográfica ganharam nova roupagem com o aumento abrupto das taxas de crescimento populacional nos países subdesenvolvidos. A mortalidade passou a cair nesses países em ritmo muito mais acelerado do que o observado no passado, com a importação maciça de uma série de tecnologias médicas e sanitárias dos países industriais (YUNES; RONCHEZEL, 1971), enquanto a natalidade parecia imóvel nesse ambiente de profundas transformações sociais.

A visão de que o crescimento demográfico exagerado traria consequências graves para as populações ganhou força num dado momento histórico. Vários trabalhos buscaram entender esta relação com base em uma perspectiva neomalthusiana. ${ }^{5}$ Autores como o norte-americano Paul Ralph Ehrlich - originalmente um biólogo que iniciou a carreira acadêmica como entomologista, mas que se tornou famoso na demografia ao publicar, em 1968, o polêmico livro The population bomb - passaram a expressar em alto e bom tom suas preocupações relacionadas com o crescimento populacional e o crescente consumo dos limitados recursos naturais disponíveis no planeta.

A temida explosão demográfica dos países subdesenvolvidos tornou-se a principal preocupação dos demógrafos por todo o mundo, que direcionaram suas atenções para as altas taxas de fecundidade e a permanência de uma estrutura etária jovem em diversos países do mundo. Os debates consideravam que o crescimento demográfico, principalmente dos países mais pobres, seria a grande ameaça à estabilidade política e econômica e à manutenção da pobreza no mundo. Diante disso, os neomalthusianos defendiam políticas

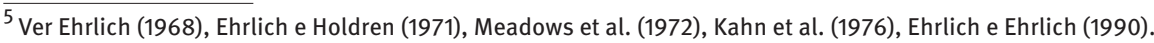


de controle populacional, incentivando a adoção de métodos contraceptivos, práticas de esterilização e aborto.

Apesar de o renascimento do malthusianismo ser fortemente relacionado ao final dos anos 1960 e início dos 1970, já existiam, anteriormente, autores e publicações defendendo este ideário, algumas vezes associado à defesa de teses eugênicas. Dois livros publicados entre as décadas de 1940 e 1950 merecem destaque: Our plundered planet, publicado por Henry Fairfield Osborn Jr. (1948), ${ }^{6}$ e Road to survival, de William Vogt (1948). ${ }^{7}$

Por outro lado, como os textos do Marquês de Condorcet já se opunham à lógica malthusiana ainda no século XVIII (ALVES, 2002), muitas vozes levantaram-se para contrapor-se à emergência do neomalthusianismo no século XX, como, por exemplo, o economista norte-americano Julian Lincoln Simon. Ele defendia a teoria, posteriormente denominada de abordagem cornucopiana, de que a capacidade de suporte à vida humana no planeta era, essencialmente, ilimitada, principalmente em função da habilidade dos humanos de desenvolverem tecnologias capazes de aumentar de forma sistemática e consistente a produção de alimentos (SIMON, 1981).

De maneira geral, a abordagem cornucopiana baseia-se na crença de que a racionalidade econômica e a eficiência tecnológica são capazes de fornecer as ferramentas necessárias tanto para produzir alimentos e outros itens essenciais em abundância quanto para resolver os problemas ambientais, promovendo a qualidade de vida das populações e garantindo, assim, a sobrevivência da espécie humana (SIMON, 1981).

Um dos principais defensores desta abordagem é a economista dinamarquesa Ester Boserup, autora de diversos livros, dos quais se destaca The conditions of agricultural growth: the economics of agrarian change under population pressure, de 1965. Nessa obra, Boserup analisa a relação entre crescimento populacional e produção de alimentos, apontando a importância que as inovações técnicas agrícolas têm desempenhado na história.

Outro livro dessa autora, Population and technological change: a study of long-term trends, publicado em 1981, teve a contribuição fundamental de ampliar seu modelo de análise para a sociedade como um todo, introduzindo na discussão sobre a relação sociedade-ambiente o conceito de economias de escala. Ainda de acordo com Boserup (1981b; 1991), diversas tecnologias só podem ser utilizadas com eficiência se a população atingir determinada densidade adequada e o crescimento demográfico tornar possível a urbanização e o processo civilizatório dela decorrente.

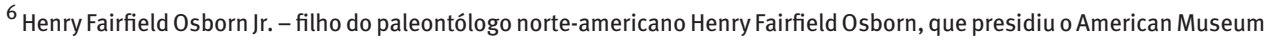
of Natural History por 25 anos e ficou famoso nas primeiras décadas do século XX por defender a eugenia e a supremacia da raça ariana - foi um biólogo norte-americano que se dedicou a estudar e defender as causas ambientais. Seus livros Our plundered planet, de 1948, The limits of the Earth, de 1953 e Our crowded planet, de 1962, são considerados alicerces conceituais da teoria neomalthusiana das décadas de 1960 e 1970.

${ }^{7}$ William Vogt foi um ecólogo norte-americano dedicado a defender a causa do controle de natalidade, especialmente das populações pobres, e a estudar as relações entre clima, população e recursos naturais na América Latina, que lançaram as bases para a publicação de seu livro Road to survival, em que ele defende a teoria de que os crescimentos econômico e das taxas de fecundidade observados no período após a Segunda Guerra Mundial ameaçavam o ambiente e a qualidade de vida das gerações futuras.
} 
A partir da década de 1970, o número médio de filhos por mulher também começou a diminuir, ainda que de forma discreta, nos países à época denominados subdesenvolvidos e, com o tempo, verificou-se que o crescimento explosivo da população, nas próximas décadas, deixaria de ser uma realidade em grande parte dos países do mundo (MARTINE, 1993; SAWYER, 1993).

Sobretudo a partir da Conferência do Cairo (1994), os embates entre os neomalthusianos e os anti-neomalthusianos perderam espaço. A mudança de paradigma e a consciência da importância dos direitos sexuais e reprodutivos ajudaram a reforçar a ideia de que 0 desenvolvimento seria o "melhor contraceptivo" (ALVES; CORREA, 2003).

A transição demográfica nos países emergentes serviu para desarmar a bomba populacional, mesmo que, de forma geral, as populações mais pobres e em maior situação de vulnerabilidade do mundo ainda exibam fecundidade em níveis superiores ao de reposição (LEE, 2003; LESTHAEGHE, 2010).

Todos estes debates não vieram acompanhados de uma discussão sistemática de cunho ambiental, embora, atualmente, fique mais fácil relacionar estes grandes embates ideológicos que marcaram a história da Demografia com o tema. Em um mundo aparentemente livre da explosão populacional, qual seriam as principais preocupações da Demografia no que diz respeito aos impactos ambientais contemporâneos? Se o ritmo de crescimento populacional tem sido freado pela transição demográfica, os problemas ambientais no mundo não deixaram de existir e têm incorporado uma nova gama de questões.

Apenas a partir da década de 1990, estudos passaram a apresentar com frequência uma visão crítica à lógica malthusiana que conecta a superpopulação à degradação ambiental em escala planetária. No Brasil, a primeira geração de demógrafos preocupados com as intercessões entre a Demografia e a questão ambiental buscou, inicialmente, incorporar o tema meio ambiente às discussões entre população e desenvolvimento (HOGAN, 2007).

O livro organizado por George Martine (1993), População, meio ambiente e desenvolvimento: verdades e contradições, reuniu os primeiros esforços de pesquisadores brasileiros que há 25 anos vêm trabalhando pela consolidação da demografia ambiental, sendo um dos mais importantes marcos na construção do campo de discussão no Brasil.

Sawyer (1993) argumentou que a questão do consumo aparece atrelada a estas primeiras discussões sobre população, desenvolvimento e ambiente. Nos anos que se seguiram, diversos trabalhos apontaram que a explosão do consumo se tornou algo muito mais preocupante do que o crescimento exagerado da população (JACKSON, 2006; HOGAN et al., 2010; ROYAL SOCIETY, 2012).

A análise do crescimento demográfico sempre será importante para as discussões ambientais em todas as escalas, desde que acompanhada de uma visão crítica que considere também outros aspectos da dinâmica demográfica, como localização espacial, estrutura etária, padrões de fecundidade e envelhecimento populacional (HOGAN, 1991; MARTINE, 1993; SAWYER, 1993). 
A localização espacial da população e os padrões recentes de urbanização não podem ser esquecidos nos debates sobre população, ambiente e consumo. As grandes mudanças testemunhadas recentemente no mundo na localização espacial das populações implicaram, necessariamente, fortes transformações nas relações do homem com o ambiente e, também, nos padrões de produção e consumo.

Segundo Martine (2007), a concentração urbana pode facilitar o enfrentamento dos problemas ambientais. Na visão do autor, se, por um lado, maior disponibilidade de terra no campo permite ganhos na produtividade agrícola e facilita a preservação dos ecossistemas, por outro, a concentração de pessoas nas cidades abre um leque de oportunidades para a universalização de serviços ambientais básicos. Martine (2007, p. 188) declara que "a densidade urbana mostra-se favorável ao crescimento econômico, ao desenvolvimento social e à redução da fecundidade, além de favorecer um uso mais eficiente dos recursos". Assim, a urbanização também é responsável por importantes impactos no crescimento populacional e na estrutura etária das populações.

As relações entre estrutura etária e as diversas formas de consumo também têm alcançado destaque na literatura recente, se mostrando um campo de estudos promissor. Segundo Campos (2014, p. 18), “os padrões de consumo das sociedades são influenciados, dentre outros aspectos, pela estrutura etária da população, traduzida em sua composição por grandes grupos etários: crianças, adultos e idosos". Conforme o autor, vale lembrar que a composição da população é, inclusive, um determinante importante para sua taxa de crescimento.

Diversos estudos vêm explorando esta intercessão entre a mudança na estrutura etária e variáveis de consumo, como os incrementos no uso de combustíveis (GRENNING; JENG, 1994), na demanda domiciliar por energia (O`NEILL; CHEN, 2002), na poluição do ar (COLE; NEUMAYER, 2004) e na demanda por automóveis (PRSKAWETZ et al., 2004), decorrentes das novas estruturas etárias marcadas pelo envelhecimento da população.

Ojima e Carvalho (2009) argumentam que, de modo geral, a transição demográfica e a consequente redução do número médio de moradores por domicílio incentivam o aumento nos fatores de impacto ambiental com a elevação do padrão de consumo.

Recentemente, a revista Demography publicou um debate interessante sobre população e ambiente entre David Lam e Stan Becker, em que a questão do consumo é destacada, apontando para a atualidade e relevância da discussão e a crescente necessidade de a Demografia brasileira se manifestar e se envolver com a mesma.

Com base em análises dos últimos 50 anos, período de intenso crescimento populacional no planeta, Lam (2011) argumenta que, apesar de todos os temores lançados nos anos 1960 (fome, exaustão de recursos naturais não renováveis e aumento da pobreza), a humanidade ampliou a produção de alimentos, reduziu o preço dos recursos e diminuiu significativamente a pobreza na maior parte do mundo em desenvolvimento. 0 autor apresenta uma visão otimista entre desenvolvimento e ambiente e espera mudanças positivas até 2050. 
No entanto, Becker (2013, p. 1231) destaca que, “embora Lam aborde de maneira breve as mudanças climáticas e a poluição, o autor não avalia vários outros problemas ambientais que estão intimamente relacionados ao rápido crescimento populacional”. Com o incremento de quatro bilhões de pessoas no planeta, Becker defende que a humanidade está consumindo recursos em um nível insustentável, causando desequilíbrios ambientais sérios, a exemplo da escassez de água subterrânea e redução do volume dos rios, utilização excessiva de combustíveis fósseis e de recursos minerais, desflorestamento e extinção de espécies. Becker ainda argumenta que a abordagem de Lam (2011) refere-se ao mundo dos humanos, negligenciando as outras espécies e demais componentes do meio natural.

Lam (2013) justifica sua visão otimista, destacando que tendências positivas são geralmente esquecidas, mesmo que se sobreponham a outras tendências negativas. Embora exista consenso sobre os desafios a serem enfrentados nas próximas décadas, o autor ressalta que, apesar de o mundo ter sobrevivido à bomba populacional, isso não significa que este caminho tenha sido fácil ou que não tenham aparecido novos desafios. 0 autor também chama a atenção para um feito impressionante: a humanidade conseguiu adicionar quatro bilhões de pessoas ao mundo e, mesmo assim, melhorar a maior parte das medidas de bem-estar, o que requer trabalho duro, engenhosidade e boas instituições. Assim, ele defende que podemos alimentar e hospedar mais três bilhões de pessoas até o final do século, sem negligenciar os desafios ambientais que Becker (2013) destacou.

No debate entre David Lam e Stan Becker, assim como em diversos estudos apresentados neste trabalho sobre população e ambiente, a questão do consumo é fundamental. 0 aumento do consumo esbarra, a todo o momento, nos limites ambientais do planeta e nas mudanças observadas na natureza. 0 crescimento econômico e o aumento geral da renda vêm sendo apresentados e defendidos, sistematicamente, como fator essencial para a expansão do acesso e do usufruto de melhores padrões de qualidade de vida e condições mais dignas por significativos contingentes populacionais que ainda não estão inseridos nos circuitos globais do consumo.

A realidade é que parte significativa da população mundial é extremamente pobre e vive em condições precárias que ameaçam a saúde e a vida, sobretudo nos países em desenvolvimento e naqueles menos desenvolvidos. A eliminação geral da pobreza é desejada e será um enorme ganho social caso se concretize. Não obstante a enorme desigualdade na distribuição de riquezas no mundo, a redução da pobreza e/ou o aumento da renda também poderão contribuir para a intensificação dos impactos ambientais caso não existam maior equilíbrio no acesso aos recursos naturais, mudança tecnológica ou transformações no tipo de consumo (SATHLER, 2012).

Por outro lado, Sawyer (2002), ao contrário das principais agências internacionais de fomento à pesquisa sobre desenvolvimento, tem assinalado que a redução da pobreza não necessariamente resultará em aumento automático da degradação ambiental no mundo. Conforme o autor, muitos trabalhos sobre a relação entre população e consumo são for- 
temente influenciados por uma visão parcialmente verdadeira, mas muito simplificada, de que a degradação ambiental é causada pelo consumo no Norte e pela pobreza no Sul.

A Demografia deve estar atenta para os novos desafios impostos pela realidade: os efeitos da transição demográfica e das alterações na composição domiciliar no ambiente; o impacto dos movimentos migratórios e das implicações ambientais da urbanização e da distribuição espacial da população; o aumento do acesso ao mercado de consumo por parte de populações antes marginalizadas; entre outras questões emergentes que merecem a atenção da comunidade científica.

Assim, existe um grande leque de oportunidades para a produção de abordagens reflexivas que buscam entender como as variáveis demográficas podem influenciar os padrões e os níveis de consumo, e como a Demografia pode contribuir para a adaptação e a mitigação dos problemas ambientais emergentes.

\section{Considerações finais}

A Demografia oferece diversas possibilidades analíticas para os estudos sobre a questão ambiental, embora haja muito que fazer em busca de uma demografia ambiental mais estruturada e influente nos círculos acadêmicos. Os estudos sobre população, espaço e ambiente devem estar pautados na integração entre os diferentes campos de conhecimento para a consolidação de uma ciência verdadeiramente interdisciplinar, que não segmente e fragmente o conhecimento e o entendimento da realidade.

Nesse contexto, as abordagens integradas sobre população, consumo e ambiente ganharam fôlego nos círculos de debates ambientais, embora ainda careçam de ampliação. De maneira geral, os estudos que lançam uma perspectiva demográfica sobre as implicações ambientais do crescimento do consumo esbarram com a constante necessidade de desmistificar qualquer associação simplista entre crescimento demográfico e degradação ambiental.

O modelo de desenvolvimento em curso não consegue estabelecer uma relação sustentável com as práticas de consumo das populações, reforçando, a cada dia, a ideia de que estamos caminhando novamente para uma situação de catástrofe, caso não haja alguma mudança social, econômica, tecnológica ou cultural relevante nas próximas décadas. Assim, não obstante o enorme volume de problemas ambientais acumulados pela sociedade e que precisam ser resolvidos, o mundo deve se preparar para enfrentar os novos desafios advindos do aumento dos níveis de renda e do consumo nas sociedades capitalistas.

No espírito do pensamento malthusiano/neomalthusiano, os fantasmas do passado parecem voltar de tempos em tempos. Após a divulgação do AR-4 pelo IPCC em 2007 e, também, de que a população mundial alcançou sete bilhões em 2011, a abordagem malthusiana foi resgatada com certa frequência por entidades não governamentais e ambientalistas.

A incorporação de temas demográficos pela sociedade e por pesquisadores de outras áreas do conhecimento, vez ou outra, resulta em visões simplistas que pou- 
co contribuem ou até atrapalham os debates sobre população e ambiente. Isso tem acontecido recorrentemente, muito mais pela elaboração de opiniões desvinculadas de uma revisão teórica consistente em Demografia do que, necessariamente, pela existência de uma escola neomalthusiana influente nos círculos acadêmicos e políticos na atualidade.

Novas abordagens que capturem as conexões da dinâmica demográfica com os padrões de produção e consumo são cada vez mais necessárias. 0 momento atual é, cada vez mais, de ação. As recentes crises financeiras, assim como os eventos climáticos e ambientais extremos dos últimos anos, lançam novos desafios que devem ser transpostos também para as discussões entre consumo e ambiente.

Nessa crescente busca por respostas, já existem sementes que apontam para formas mais adequadas de desenvolvimento e de outra concepção de qualidade de vida e felicidade, buscando formas de produção e consumo mais inteligentes, menos exclusão e desigualdade, assim como a redução das interferências negativas no frágil equilíbrio que garante a existência da vida.

\section{Referências}

ALVES, J. E. D. A polêmica Malthus versus Condorcet reavaliada à luz da transição demográfica. Rio de Janeiro: Ence/IBGE, 2002 (Texto para discussão, n. 4).

População, bem-estar e tecnologia: debate histórico e perspectivas. Multiciência, v. 6, n. 1, p. 1-24, 2006.

ALVES, J. E. D.; CORRÊA, S. Demografia e ideologia: trajetos históricos e os desafios do Cairo+10. Revista Brasileira de Estudos de População, v. 20, n. 2, p. 129-156, 2003.

AMANN, C.; BRUCKENER, W.; FISCHER-KOWALSKI, M.; GRÜNBÜHEL, C. Material flow accounting in Amazonia: a tool for sustainable development. Social Ecology Working Paper, n. 63, p. 2-25, 2002.

ANDERTON, D. L.; ANDERSON, A. B.; OAKES, J. M. et al. Environmental equity: the demographics of dumping. Demography, n. 31, n. 2, p. 229-248, 1994.

ARROW, K.; BOLIN, B.; COSTANZA, R.; DASGUPTA, P.; FOLKE, C.; HOLLING, C. S.; JANSSON, B.; LEVIN, S.; MALER, K. G.; PERRINGS, C.; PIMENTEL, D. Economic growth, carrying capacity, and the environment. Science, n. 268, p. 520-521, 1995.

BECKER, S. Has the world really survived the population bomb? (Commentary on "how the world survived the population bomb: lessons from 50 years of extraordinary demographic history"). Demography, v. 50, n. 6, p. 2173-2181, 2013.

BILSBORROW, R. E.; DELARGY, P. Land use, migration and natural resource deterioration: the experience of Guatemala and the Sudan. Population and Development Review, v. 16 (supplement), p. 125-147, 1991.

BILSBORROW, R. E.; STUPP, P. Population dynamics, land use change and deforestation in Guatemala. In: PEBLEY, A.; BIXBY, L. R. (Orgs.). Population and development in the Isthmus of Central America. Santa Barbara, CA: Rand Corporation, 1997. p. 581-623.

BIRDSALL, N. Another look at population and global warming. Washington: Policy Research Working Papers, 1992. 
BONGAARTS, J. Population growth and global warming. Population and Development Review, v. 18, n. 2, p. 299-319, 1992.

BOSERUP, E. The conditions of agricultural growth: the economics of agrarian change under population pressure. London: George Allen \& Unwin, 1965.

Population and technological change: a study of long-term trends. Chicago: The University of Chicago Press, 1981a.

Environment, population, and technology in primitive societies. Population and Development Review, v. 2, n. 1, p. 21-36, 1981b.

Causes and effects of disequilibria in food production. In: GENDREAU, F.; MEILLASSOUX, C.; SCHLEMMER, B.; VERLET, M. (Orgs.). Les spectres de Malthus: déséquilibres alimentaires, déséquilibres démographiques. Paris: EDI/ORSTOM/CEPED, 1991. p. 33-40.

BRINGEZU, S.; FISCHER-KOWALSKI, M.; KLEIN, R.; PALM, V. Regional and national material flow accounting: from paradigm to practice of sustainability. Leiden: Leiden University, 1997.

CAMPOS, M. B. Por uma reflexão demográfica das mudanças ambientais no Brasil. Revista Espinhaço, v. 3, n. 1, p. 17-23, 2014.

CARRABINE, E. Consumo. In: SCOTT, J. (Org.). Sociologia: conceitos-chave. Rio de Janeiro: Zahar, 2010.

COLE, M. A.; NEUMAYER, E. Examining the impact of demographic factors on air pollution. Population and Environment, v. 26, n. 1, p. 5-21, 2004.

CURRAN, S. R.; DE SHERBININ, A. Completing the picture: the challenges of bringing "consumption" into the population-environment equation. Population and Environment, v. 26, n. 2, p. 107-131, 2004

DAVIS, K. Population and resourses: fact and interpretation. In: DAVIS, K.; BERNSTAN, M. S. (Orgs.). Resourses, environment and population: presente knowleged, future options. New York: Oxford University Press, 1991. p. 1-24.

DENATRAN. Banco de dados e de informações. Disponível em: 〈http://www.denatran.gov.br/. 2011>. Acesso em: 4 jul. 2014.

EHRLICH, P. R. The population bomb. New York: Oxford University Press, 1968.

EHRLICH, P. R.; HOLDREN, J. P. Impact of population growth. Science, n. 171, p. 1212-1217, 1971.

EHRLICH, P. R.; EHRLICH, A. H. The population explosion. London: Hutchinson, 1990.

EUROSTAT. Economy-wide material flow accounts and derived indicators. A methodological guide. Luxemburg: Europe Statistical Office of the European Union, 2001.

FEATHERSTONE, M. Cultura de consumo e pós-modernismo. São Paulo: Studio Nobel, 1995.

FREIRE DE MELLO, L.; HOGAN, D. J. População, consumo e meio ambiente. In: HOGAN, D. J. (Org.). Dinâmica populacional e mudança ambiental: cenários para o desenvolvimento brasileiro. Campinas: Núcleo de Estudos de População - Nepo/Unicamp, 2007. p. 59-72.

FREIRE DE MELLO, L. População, consumo e mudança climática. In: HOGAN, D. J.; MARANDOLA JR., E. (Orgs.). População e mudança climática: dimensões humanas das mudanças ambientais globais. Campinas: Núcleo de Estudos de População - Nepo/Unicamp, Brasília: UNFPA, 2009.

GARDNER, G.; ASSADOURIAN, E.; SARIN, R. O Estado do consumo hoje. In: WORLDWATCH INSTITUTE. Estado do mundo, 2004: estado do consumo e o consumo sustentável. Salvador: UMA Editora, 2004. 
GONÇALVES, L. A. Direitos sociais, cidadania, política e justiça. Rio de Janeiro: Sinergia, 2013. GRENNING, L. A.; JENG, T. H. Lifecycle analysis of gasoline expenditure patterns. Energy Economics, v. 16, n. 3, p. 217-228, 1994.

HABERL, H.; FISCHER-KOWALSKI, M.; KRAUSMANN, F.; WEISZ, H.; WINIWARTER, V. Progress towards sustainability? What the conceptual framework of material and energy flow accounting (MEFA) can offer. Land Use Policy, v. 21, n. 3, p. 199-213, 2004.

HAMMER, M.; HUBACEK, K. Material flows and economic development. Material flow analysis of the Hungarian economy. Luxemburg: International Institute for Applied Systems Analysis (IIASA), 2002.

HARDOY, J.; PANDIELLA, G. Urban poverty and vulnerability to climate change in Latin America. Environment and Urbanization, v. 21, n. 1, p. 203-224, 2009.

HARPER, S. Migration and global environmental change. Oxford: Oxford University Press, 2001.

HINTERBERGER, F.; GILJUM, S.; HAMMER, M. Material flow accounting and analysis (MFA). A valuable tool for analyses of society-nature interrelationships entry prepared for the internet encyclopedia of ecological economics, 2003. p. 1-19.

HOGAN, D. J. Dinâmica demográfica e poluição ambiental. Textos Nepo, v. 1, n. 12, p. 1-145, 1987. . População e meio ambiente. Textos Nepo, v. 16, n. 1, p. 1-80, 1989.

. Demographic aspects of global environmental change: what is Brazil's contribution? In: HOGAN, D.; TOLMASQUIM, M. Human dimensions of global environmental change. Rio de Janeiro: Academia Brasileira de Ciências, 2001.

. População e meio ambiente: a emergência de um novo campo de estudos. In: HOGAN, D. J. Dinâmica populacional e mudança ambiental: cenários para o desenvolvimento brasileiro. Campinas: Nepo/Unicamp, 2007.

. Demographic dynamics and environmental change in Brazil. Ambiente e Sociedade, n. 9, p. 43-73. 2001. Disponivel em: 〈http://www.scielo.br/scielo. php?script=sci_arttext\&pid $=$ S1414753X2001000900004\&lng $=$ en\&tlng $=$ en.10.1590/S1414-753X2001000900 004 . Acesso em: 5 jul. 2014.

HOGAN, D. J.; MARANDOLA JR., E.; OJIMA, R. População e ambiente: desafios à sustentabilidade. São Paulo: Blucher, 2010.

HOLDEN, E. Ecological footprints and sustainable urban form. Journal of Housing and the Built Environment, v. 19, n. 1, p. 91-109, 2004.

HUNTER, L. M. The association between environmental risk and internal migration flows. Population and Environment, v. 19, n. 3, p. 247-277, 1998.

IPCC. Climate Change 2013. The physical science basis. Contribution of working group I to the fifth assessment report of the Intergovernmental Panel on Climate Change. Cambridge; United Kingdom; New York: Cambridge University Press, 2013.

JACKSON, T. Beyond the 'wellbeing paradox': wellbeing, consumption growth and sustainability. CES Working Paper, v. 6, n. 6, p. 4-31, 2006.

JOHNSON, A. G. Dicionário de sociologia: guia prático da linguagem sociológica. Rio de Janeiro: Zahar, 1997.

KAHN, H.; BROWN, W. L. Martel the next 200 years. New York: Books on Tape, 1976.

KATES, R. W. Population and consumption: what we know, what we need to know. Environment, V. 42 , n. 3, p. 10-19, 2000. 
KEMPF, H. How the rich are destroying the Earth. Vermont: Chelsea Green Publishing, 2008.

KEYFITZ, N. Seven ways of causing the less developed countries population problem to disappear - in theory. European Journal of Population, v. 8, n. 1, p. 149-67, 1992.

KUMAR, M. Consumption in the age of consumer capitalism: the question of consumer sovereignty. In: INTERNATIONAL CONFERENCE ON CONSUMERISM (ICC2013). Proceedings... Berlin, 2013.

LAM, D. How the world survived the population bomb: lessons from 50 years of extraordinary demographic history. Demography, v. 48, n. 4, p. 1231-1262, 2011.

. Reply to Stan Becker, "Has the World Really Survived the Population Bomb? (Commentary on "How the World Survived the Population Bomb: Lessons from 50 Years of Extraordinary Demographic History”)”. Demography, v. 50, n. 6, p. 2183-2186, 2013.

LEE, R. The demographic transition: three centuries of fundamental change. Journal of Economic Perspectives, v. 17, n. 4, p. 167-190, 2003.

LESTHAEGHE, R. The unfolding story of the second demographic transition. In: CONFERENCE ON FERTILITY IN THE HISTORY OF THE 20TH CENTURY - TRENDS, THEORIES, PUBLIC DISCOURSE, AND POLICIES. Berlin, 2010.

MALTHUS, T. R. Ensaio sobre população. São Paulo: Abril Cultural, 1983.

MARANDOLA JR., E.; HOGAN, D. J. Em direção a uma demografia ambiental? Avaliação e tendências dos estudos de população e ambiente no Brasil. Revista Brasileira de Estudos de População, v. 24, n. 2, p. 191-223, 2007.

MARTINE, G. (Org.). População, meio ambiente e desenvolvimento: verdades e contradições. Campinas: Ed. da Unicamp, 1993.

. O lugar do espaço na equação população/meio ambiente. Revista Brasileira de Estudos de População, v. 24, n. 2, p. 181-190, 2007.

MARTINE, G.; SCHENSUL, D. The demography of adaptation to climate change. New York; London; Mexico City: UNFPA; IIED; El Colegio de México, 2013.

MCCRACKEN, G. Cultura e consumo: novas abordagens ao caráter simbólico dos bens e das atividades de consumo. Rio de Janeiro: Mauad, 2003.

MEADOWS, D. L. Limites do crescimento: um relatório para o Projeto do Clube de Roma sobre o dilema da humanidade. São Paulo: Perspectiva, 1972.

MINDELL, D. The science and technology of World War II. Household behaviour and the environment reviewing the evidence. Paris, France, 2009. Disponivel em: 〈http://www.learnnc. org/lp/pdf/the-science-and-technology-p6002.pdf>. Acesso em: 4 jul. 2014.

MYERS, N.; Kent, J. New consumers: the influence of affluence on the environment. Proc. Natl. Acad. Sciences USA, v. 100, n. 8, p. 4963-4968, 2003.

MYERS, N. Consumption in relation to population, environment and development. The Environmentalist, v. 17, n. 1, p. 33-44, 1997.

OECD. Annual report 2008. Disponível em: 〈www.oecd.org/newsroom/40556222.pdf〉. Acesso em: 4 jul. 2014.

OJIMA, R.; CARVALHO, R. L. Gênero, família e meio ambiente: limites e perspectivas para o campo dos estudos de população. In: SEMINÁRIO AVANÇOS E DESAFIOS NO USO DO CONCEITO DE GÊNERO NOS ESTUDOS POPULACIONAIS. Rio de Janeiro, 22 e 23 de outubro de 2009.

O’NEILL, B. C.; MACKELLAR, F. L.; LUTZ, W. Population and climate change. Cambridge: Cambridge University Press, 2001. 
O'NEILL, B. C.; CHEN, B. S. Demographic determinants of household energy use in the United States. Population and Development Review, v. 28, n. 1, p. 58-88, 2002.

ONU. Relatório de Desenvolvimento Humano 2011. Nova York: PNUD, 2011.

OSBORN JR., H. F. Our plundered planet. Boston: Little, Brown \& Co, 1948.

PARSONS, T. Structure and process in modern society. New York: Free Press, 1960.

PATERSON, M. Consumption and everyday life. London: Routledge, 2006.

PEBLEY, A. R. Demography and the environment. Santa Monica, v. 35, n. 4, p. 377-389, 1998.

PRINCEN, T.; MANIATES, M.; CONCA, K. Confronting consumption. Massachusetts: The MIT Press, 2002.

PRSKAWETZ, A.; LEIWEN, J.; O’NEILL, B. C. Demographic composition and projections of car use in Austria. Vienna Yearbook of Population Research, v. 2, n. 1, p. 175-201, 2004.

RCEP. Demographic change and the environment. London: Twenty-nine report, 2011.

ROYAL SOCIETY. People and the planet - The Royal Society Science Policy Centre report 01/12. London: The Royal Society, 2012.

SATHLER, D. População, consumo e ambiente: contribuições da Demografia para a questão ambiental. In: XVIII ENCONTRO NACIONAL DE ESTUDOS POPULACIONAIS. Anais... Águas de Lindóia: Abep, 2012. Disponivel em: 〈www.abep.nepo.unicamp.br/xviii/anais/files/ST36[810] ABEP2012.pdf〉. Acesso em: 4 jul. 2014.

SAWYER, D. População, meio ambiente e desenvolvimento no Brasil. Brasília: ISPN, 1993.

Consumption patterns and environmental impacts in a global socioecosystemic perspective. In: HOGAN, D. J. (Org.). Human dimensions of global environmental change. Rio de Janeiro: Academia Brasileira de Ciências, 2001.

. Population and sustainable consumption in Brazil. In: HOGAN, D. J.; BERQUÓ, E.; COSTA, H. S. M. (Orgs.). Population and environment in Brazil: Rio+10. Campinas: CNPD/ Abep/ Nepo-Unicamp, 2002.

SENNETT, R. A cultura do novo capitalismo. Rio de Janeiro: Record, 2006.

SIMON, J. L. The ultimate resource. Princeton: Princeton University Press, 1981.

SLATER, D. Cultura do consumo \& modernidade. São Paulo: Nobel, 2001.

SPAARGAREN, G. Lifestyles, consumption and the environment: the ecological modernisation of domestic consumption. In: MOL, A. P. J.; SONNENFELD, D. A. (Orgs.). Ecological modernisation around the world: perspectives and critical debates. Londres: Frank Cass Publishers, 2000.

. Ecological modernization theory and domestic consumption. Journal of Environmental Policy \& Planning, v. 2, n. 4, p. 323-335, 2000.

Sustainable consumption: a theoretical and environmental policy perspective. Society \& Natural Resources, v. 16, n. 8, p. 687-701, 2003.

STERN, D. I. The rise and fall of the environmental Kuznets Curve. World Development, v. 32, n. 8, p. 1419-1439, 2004.

STERN, P. C.; DIETZ, T.; RUTTAN, V. W.; SOCOLOW, R. H.; SWEENEY, J. L. Environmentally significant consumption. Research directions. Washington: National Academy Press, 1997.

THORGERSON, J.; OLANDER, F. Human values and the emergence of a sustainable consumption pattern: a panel study. Journal of Economic Psychology, v. 23, n. 1, p. 605-630, 2002. 
UNEP - United Nations Environment Programme. Sustainable consumption - A global status report. Lund: Lund University, Institute for Industrial Environmental Economics (IIEE), 2002.

Assessing the environmental impacts of consumption and production: priority products and materials. 2010.

UN-HABITAT. Istanbul declaration on human settlements. 1996. Disponivel em: 〈http://ww2. unhabitat.org/declarations/ch-1a. htm>. Acesso em: 6 jul. 2014.

WACKERNAGEL, M.; ONISTO, L.; BELLO, P.; LINARES, A. C.; FALFAÉN, I. S. L.; GARCIA, J. M.; GUERRERO, A. I. S.; GUERRERO, M. G. S. National natural capital accounting with the ecological footprint concept. Ecological Economics, v. 29, n. 1, p. 375-390, 1999.

WACKERNAGEL, M.; REES, W. Our ecological footprint. Gabriola Islands: New Society Publishers, 1988.

WILK, R. Emulation, imitation and global consumerism. Organization and Environment, v. 11, n. 3, p. 314-333, 1998.

. Consumption, human needs, and global environmental change. Global Environmental Change, v. 12, n. 1, p. 5-13, 2002.

WOOD, C. H.; CARVALHO, J. A. M. The demography of inequality in Brazil. Cambridge: Cambridge University Press, 1988.

YUNES, J.; RONCHEZEL, V. S. C. Evolução da mortalidade geral, infantil e proporcional no Brasil. Revista de Saúde Pública, v. 8, n. 1, p. 3-48, 1971.

\title{
Sobre os autores
}

Leonardo Freire de Mello é doutor em Demografia pela Universidade Estadual de Campinas Unicamp. Professor adjunto do Bacharelado em Planejamento Territorial e do Programa de Pós-Graduação em Planejamento e Gestão do Território da Universidade Federal do ABC - UFABC.

Douglas Sathler é doutor em Demografia pela Universidade Federal de Minas Gerais - UFMG. Professor adjunto II da Universidade dos Vales do Jequitinhonha e Mucuri - UFVJM.

\section{Endereço para correspondência}

\author{
Leonardo Freire de Mello \\ Rua Arcturus, 3, bloco Delta, sala 387, Jardim Antares \\ 09606-070 - São Bernardo do Campo-SP, Brasil \\ Douglas Sathler \\ Rodovia MGT 367, km 583, 5.000, Alto da Jacuba \\ 39100-000 - Diamantina-MG, Brasil
}

\section{Abstract \\ Environmental demography and the emergence of population and consumption studies}

In recent years, Demography has devoted more effort to assess environmental issues based on interdisciplinary approaches. Demography's incorporation of environmental issues is consistent with what can be observed in almost every field of knowledge, with a clear increase in the 
concerns related to sustainability of the planet. At the center of today's environmental debate the problems caused by the imbalance between consumption, environment and population deserve more attention. First, this study offers some reflections on the recent consolidation of environmental demography, seeking to evaluate the paths not yet taken, and the growth possibilities for studies on population, consumption and environment. The paper then presents an overview of the approaches offered by the social sciences on consumption, focusing on how these studies have incorporated environmental issues. Finally, the study explores the inclusion of this topic - consumption and environment - in Demography, overcoming of old paradigms and revealing new challenges. The reflections in this work show that the demographic perspective on the environmental implications of consumption growth come up against the constant need to demystify any simplistic association between demographic growth and environmental degradation. Currently, the consumption boom is increasingly seen as something much more concerning than the population overgrowth.

Keywords: Population. Environment. Consumption.

\section{Resumen}

La demografía ambiental y la emergencia de los estudios sobre población y consumo

En los últimos años la demografía ha dedicado más atención a los abordajes interdisciplinarios sobre la cuestión ambiental. La incorporación de la temática a esta disciplina está en sintonía con lo que se ha observado en prácticamente todos los campos del conocimiento, ante la clara valorización de las preocupaciones relacionadas con la sustentabilidad en el planeta. En el centro de las principales discusiones ambientales actuales merecen cada vez mayor atención los problemas generados por los desequilibrios entre el consumo, el ambiente y la población. En una primera parte, este artículo ofrece una serie de reflexiones sobre la consolidación reciente de la demografía ambiental, procurando evaluar los caminos aún no transitados y las posibilidades de crecimiento de los estudios sobre población, consumo y ambiente. Después se presenta una revisión de los abordajes sobre el consumo que ofrecen las ciencias sociales, investigando el modo en que estos estudios han incorporado la cuestión ambiental. Finamente, se explora la inserción del tema del consumo y el ambiente en la demografía, revelando la superación de viejos paradigmas y el surgimiento de nuevos desafíos. Las reflexiones que se realizan en este estudio demuestran que los trabajos que proponen una perspectiva demográfica sobre las implicaciones ambientales del crecimiento del consumo tropiezan con la constante necesidad de desmitificar cualquier asociación simplista entre crecimiento demográfico y degradación ambiental. Actualmente la explosión del consumo es vista, cada vez más, como algo mucho más temido que el crecimiento exagerado de la población.

Palabras clave: Población. Ambiente. Consumo.

Recebido para publicação em 06/10/2014 Recomendado para publicação 13/02/2015 Aceito para publicação em 16/08/2015 